\title{
PROPOS SUR LA RÉGENÉRATION DU CHÊNE DANS L'OUEST
}

La Revue Forestière a publié récemment un très intéressant article de M. le Conservateur Lesage, qui met en évidence la part prépondérante que doit avoir l'intervention de l'homme dans la régénération du hêtre en Normandie.

Cet article nous a suggéré quelques réflexions et décidé à exposer notre point de vue en ce qui concerne le chêne du secteur atlantique, chêne qui, d'ailleurs, dans notre esprit, est intimement lié à la présence du hêtre.

L'on pourrait presque penser que l'un des plus gros défauts du Français est d'être trop intelligent, ce qui l'amène à négliger, fort de cet instrument incomparable, les autres qualités moins brillantes qui sont cependant indispensables pour s'assurer un succès complet. Il a le premier érigé en théorie la méthode des ensemencements naturels, il a été instinctivement très séduit par cette idée qui semblait l'autoriser à diminuer considérablement la part de l'effort humain, ce qui correspond bien à son tempérament, et aussi les dépenses à engager, ce qui cadre bien, pour le forestier d'Etat, avec la pénurie à peu près chronique de crédits dans laquelle il se trouve.

On a ainsi l'impression qu'il y a quelques années, le forestier, dans les futaies de chênes de l'Ouest, apportait toutes ses facultés à marquer à propos ses coupes de régénération, à les espacer convenablement, qu'il dissertait volontiers à perte de vue sur l'éclairement de la coupe d'ensemencement, sur la cadence et le nombre des secondaires, mais que l'on. citait presque pour mémoire seulement des crochetages à exécuter au cours de la régénération. En un mot, la réussite croyait-on, était entièrement entre les mains du " bon marteau 》) et le marteau dans ce cas est forcément commandé par une bonne intelligence, mais la part de l'intervention du travail de l'homme était considérée comme nulle ou presque.

Depuis cent ans que cette méthode est appliquée, il est permis d'en établir le bilan à l'égard du chêne de l'Ouest.

Les peuplements nés au siècle dernier sont presque toujours complets et devaient présenter au départ un nombre très élevé de tiges 
si l'on en 'juge par la hauteur, la reçtitude et la propreté des fûts, par contre ceux qui sont nés depuis le début de ce siècle sont beaucoup plus irréguliers. S'il y a de très belles taches, il est rare qu'il y ait vraiment de très beaux peuplements. Trop confiant dans sa brillante trouvaille, le forestier s'est endormi, n'a plus fait travailler manuellement et n'a pas vu que la forêt voyait son potentiel diminuer à chaque coupe définitive.

Appliquée telle qu'elle était enseignée, la méthode des ensemencements naturels donnait à quelques variantes près les résultats suivants pris parmi les plus typiques.

Fn mettant les choses au mieux, si le peuplement à régénérer était encore bien vivant, si le sol était naturellement bien assaini, si les arbres ne présentaient pas un enracinement trop superficiel, si enfin les glandées, comme il est normal sous ce climat. ne se faisaient pas attendre plus de 4 ans, si, si, si... de belles taches de semis venaient rapidement sous les semenciers et pouvaient couvrir en fin de compte 1 les $3 / 4$ de la surface. Sur le dernier quart, les semis étaient soit saccagés par les vidanges, avec des chemins très nombreux formant d'énormes “ pattes d'oie » vers les sorties les plus faciles, soit inexistants et remplacés par les fougères ou les ronces qui les avaient étouffés sitôt nés. Les fourrés issus d'une telle régénération n'étaient guère parfaits que sur les deux tiers de la surface parce qu'en bordure des petits vides les jeunes arbres forment forcément " pommier ».

Dans les cas moins favorables, tous les intermédiaires se rencontrent entre cette situation, considérée comme très satisfaisante et la catastrophe totale, c'est-à-dire la définitive faite de guerre lasse au bout d'une vingtaine d'années sur une pelouse de graminées, une mer de fougères, de ronces ou de blancs. Sur les plateaux mal drainés naturellement, le jonc faisait son apparition rapidement après l'ensemencement, la gigantesque pompe formée par le peuplement n'étant plus suffisante pour éliminer l'eau superficielle. La régénération dans ce cas là n'est pas nulle, il y a des plants un peu partout, mais jamais assez nombreux pour former, malgré un bourrage de blancs dans la jeunesse, l'origine d'un beau peuplement à fûts propres. Dans les parties. exposées aux grands vents, les 'feuilles étaient régulièrement balayées et les glands gelaient faute de ce matelas protecteur que la nature leur fait cependant tomber dessus, passé le coup de fouet de nitrification décrit par M. l'Ingénieur DuCHAUFOUR après l'éclairement brutal de l'ensemencement, sans que l'on ait pu en profiter, la partie était perdue, et le passage des secondaires n'était plus qu'une récolte et seulement un simulacre de culture.

Il faut bien reconnaitre que si la nature nous est livrée admirablement agencée par son Créateur, les conditions indispensables d'une vraie régénération naturelle guidée par l'homme sont bien ra- 
rement réunies et c'était trop lui demander que de remplir au moment voulu toutes les conditions que nécessite la régénération.

Restaient bien les plantations pour remédier à ces situations à moitié bonnes, ou très mauvaises et c'est ce qui fut fait lorsque les crédits étaient suffisants. Mais il y a peu de plantations qui soient aussi onéreuses et il ne faut se faire aucune illusion, les résultats à attendre de 4000 ou même de 5000 plants à l'ha ne seront jamais comparables à ceux d'un semis pouvant donner 20000 brins et plus sur la même surface.

Ces plantations sont parmi les onéreuses, en effet: sur ces bons sols, les blancs, fougères, ronces, genêts ont formé, pendant les 20 ans qu'a duré la régénération plus ou moins manquée, un fouillis inextricable dans lequel l'ouverture des bandes de plantation coûte très cher, et s'agissant essentiellement d'une essence de lumière, le chêne, il ne peut être question ici d'accepter le passage du mort bois pour revenir à l'essence précieuse introduite sous son couvert. Le but poursuivi étant la production de grumes propres sur une bonne longueur, la densité de plantation ne peut être inférieure à 4 à 5000 à l'ha, ce qui est cher, et enfin le chêne poussant très lentement dans la jeunesse, c'est pendant 6 ou 7 ans au moins qu'il faudra inlassablement dégager avec souvent deux passages annuels si la fougère est abondante. Et il faut ajouter que si le travail est cher, i! est aussi extrêmement délicat, car il ne peut être appliqué brutalement sur toute la surface puisqu'il existe des taches, que l'on peut vraiment qualifier de naturelles qu'il faut soigneusement contourner.

Il est intéressant de chiffrer la dépense d'un semblable travail, ou plutôt de constater, une fois l'opération tout à fait terminée, ce qu'elle a coûté, car les devis en cette matière sont toujours insuffisants. Le dépouillement des calepins de contrôle de deux parcelles en forêt de Bercé et de Perseigne donne les moyennes suivantes à l'hectare, pour des régénérations qui couvraient effectivement la moitié de la surface environ:

- Cloisonnement des fourrés par une ligne de $\mathrm{I} m$ de large tous les $100 \mathrm{~m}$ pour s'y reconnaitre et les rendre. accessibles

- Ouverture de lignes de plantations ............. $9000 \mathrm{~F}$

- Plantation de 4000 plants $(2 / 3$ chênes, $1 / 3$ hêtres $)$ à l'ha réel par potets de $0,20 \times 0,20 \times 0,20$, prix des plants compris

- Entretien pendant 7 ans (regarnis $30 \%, 2$ passages par an les 3 premières années) 
Cette somme capitalisée à $2 \%$ pendant 200 ans, révolution normale de ces peuplements, représente $3570000 \mathrm{~F}$.

Une régénération médiocre, complétée par plantation est donc très onéreuse et, répétons-le, les résultats, à cause du décalage des âges entre les premiers semis et les plants plantés, comme d'un nombre toujours insuffisant de tiges à l'hectare ne seront jamais comparables à ceux d'un fourré dense de semis de même âge à peu près, couvrant toute la surface.

La méthode des régénérations naturelles, telle quelle, n'est donc pas satisfaisante. Les deux mots accolés, naturelle et méthode, paraissent d'ailleurs assez contradictoires, il semble qu'il aurait fallu au moins ajouter un mot, méthode par régénération naturelle guidée par exemple, mais ne vaut-il pas mieux, avec les moyens dont nous disposons maintenant une méthode de régénération par semis provoqués?

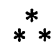

Il est curieux de constater que pendant ces mêmes années où le forestier mettait au point une méthode par laquelle, somme toute, il s'effaçait beaucoup devant les éléments naturels, les agriculteurs, issus de la même formation, s'ingéniaient au contraire à tirer du sol le plus grand profit en interférant sur lui au maximum par un travail toujours plus intensif jusqu'à arriver, au lendemain de la dernière guerre, dans certaines régions, à doubler en Io ans le rendement du blé.

Expliquant il y a quelques années à un agriculteur averti la méthode classique de régénération en forêt de Bercé, celui-ci nous en proposait tout de suite une, à sa manière... il voyait des coupes définitives sur quelques hectares, à culée noire, ce qui se fait déjà en Bercé, avec épandage d'engrais, un labour à la charrue à disques, hersage, roulage, etc... semis de glands au semoir à $0,25 \mathrm{~m}$ en tous sens et entretien à la bineuse à blé couvrant 2 ou $3 \mathrm{~m}$ d'ún seul coup, en somme quelque chose de très comparable à la culture du mais-grain qui s'est tellement développée ces années dernières, il chiffrait en gros la dépense, entretien mécanique pendant plusieurs années compris, à $50000 \mathrm{~F}$ à l'ha, la trouvant bien raisonnable comparée à une récolte escomptée de $500 \mathrm{~m}^{3}$ à $8000 \mathrm{~F}$ par exemple, soit 4 millions, oubliant seulement de capitaliser pendant 200 ans ces $50000 \mathrm{~F}$ puisque la récolte ne vient pas ici dans l'année même, mais 200 ans après en moyenne!

Sans être aussi révolutionnaire, la méthode instaurée dans l'Inspection du Mans sous l'impulsion dès I943 de M. le Conservateur VINEY complète seulement la méthode classique par une importante intégration au sol de travail humain.

Nous nous permettons de, l'exposer brièvement, ses résultats semblant devoir être très prometteurs, négligeant volontairement 
pour alléger le texte toutes les considérations classiques de la méthode, connues de tous, précisant seulement que toutes les observations et expérimentations des forestiers d'autrefois sur l'éclairement des coupes, le choix des sujets, etc... gardent toute leur valeur et qu'il faut continuer inlassablement à les compléter. Ce n'est que pour bien concrétiser l'état d'esprit d'une époque que nous avons pu paraitre précédemment ironiser un peu à propos des discussions sur ces sujets des forestiers d'alors.

Ceci étant, le but que l'on se propose est simple, il s'agit d'obtenir, sous des porte-graines naturels, des semis provoqués sur un sol réceptif et de faire en sorte que ces semis demeurent en place jusqu'à la coupe définitive sans être détruits par les débardages.

Et d'abord, il faut poser comme principe que la régénération devra être entreprise sur des peuplements encore bien vivants, ceci, qui parait pourtant évident - car un mourant ne peut prétendre se reproduire, qu'il soit du règne végétal ou animal — n'a pas été toujours reconnu. C'est ainsi que M. Potel (I) prétendait que les vieilles futaies, 300 ans, présentaient les glandées les plus fréquentes et que le sol y était plus particulièrement réceptif. Or, dans la Sarthe, les ensemencements entrepris depuis une vingtaine d'années dans des peuplements un peu décrépis naturellement, ou du fait des circonstances de guerre, par bombardements, ne donnent que des résultats très médiocres alors que les futaies en pleine vigueur des cantons de Croix-Veneur et des Hirondelles, par exemple à Bercé, donnent des tapis de semis superbes. Il s'agit là d'un problème d'aménagement. Il faut une souplesse suffisante pour que des révisions d'aménagement fréquentes permettent de classer dans le quartier de régénération les parcelles en fonction de leur vigueur et non forcément d'après leur âge, car dans une grande forêt, même apparemment homogène, le sol ne peut partout pousser le chêne jusqu'à un grand âge. Sur son champ d'un hectare, le cultivateur connait les " ronds " où les céréales seront hauts, où les betteraves flétriront et chaque fois qu'ils seront assez grands, il les traite à part ; pourquoi voudrions-nous que sur 2 ou 3000 ha le sol soit partout capable de faire pousser des chênes jusqu'à 220 ans par exemple? Ceci condamne d'une manière à peu près absolue la méthode des affectations permanentes.

Abordant donc la régénération d'une parcelle encore vivante, il parait absolument nécessaire l'année de l'exploitation de la coupe d'ensemencement d'étudier le réseau d'assainissement, pour le rendre capable de remplir son rôle. Dans les vieilles forêts, il suffira la plupart du temps de curer des fossés existants, car ce problème a été en général remarquablement résolu autrefois. Si le réseau parait insuffisant, il faut le compléter, c'est essentiel et il faudrait

(I) Du traitement de futaie par le mode dit à « tire et aire $>$. Berger-Levrault, 1925 . 
avoir le courage de ne pas commencer une régénération si l'on n'a pas les moyens d'exécuter ces travaux. Cette précaution permet au. moins d'éviter presque à coup sûr l'une des causes d'échecs décrits précédemment.

L'une des innovations de la coupe d'ensemencement est de compartimenter la parcelle à régénérer d'un quadrillage de bandes de $3 \mathrm{~m}$ de large où tous les arbres sont à marquer en abandon et à extraire à culée noire pour former tous les 100 ou $150 \mathrm{~m}$, des chemins de vidange qui seront obligatoires jusqu'à la définitive. Aucune régénération n'est attendue sur ces chemins qui devront subsister dans le fourré pour former un véritable cloisonnement qui permettra de les visiter et de les entretenir et plus tard de sortir sans dommage les premiers produits des éclaircies. En terrain relativement plat, ce quadrillage peut être géométrique, s'il y a du relief, les chemins devront en tenir compte et donc être étudiés et jalonnés avant le martelage. ·

Les grumes, lors des exploitations, devront être diablées et amenées sur ces chemins au plus court en évitant autant que faire se peut les taches de semis, puis sorties hors de la parcelle. Une clause du cahier des charges devra prévoir l'obligation de leur usage unique. Mais prévoir est facile, ce qui l'est moins, c'est d'exiger que cette clause soit respectée! Les débardeurs cherchent instinctivement à ne passer jamais deux fois à la même place pour éviter l'ornière du précédent passage et leurs traces se. rejoignent seulement en " patte d'oie " vers les sorties les plus faciles. Le sol est ainsi partout tassé et les taches de semis saccagées. Il faut leur donner dès le début un réseau suffisant, mais s'y tenir.

Au mois de septembre suivant la sortie des derniers bois de l'ensemencement, le sol doit être systématiquement travaillé par passage d'une charrue à disques, ou d'un simple covercrop, par bandes de $2 \mathrm{~m}$ tous les $2 \mathrm{~m}$, ou mieux en plein. Ceci suppose bien entendu que le cahier des charges a prévu l'extraction de tout le sous-étage à la houe et est rendu plus facile là où, comme à Bercé, les abandons sont eux-mêmes exploités à culée noire.

La découverte, qui n'est pourtant pas toute récente, des instruments à disques permet partout de réaliser ce travail qui était autrefois difficile avec les machines à socs ou à dents qui cassaient dans les racines superficielles des hêtres notamment. Les rotavators à cet égard sont désavantagés par rapport aux instruments à disques, leur utilisation est possible, mais il y aura forcément des dents cassées.

Il n'est pas utile que ce travail du sol plutôt que labour retourne complètement la terre, le but est de rendre la surface du sol rugueuse pour favoriser la germination et le premier accrochage de glands et surtout retenir les feuilles qui sans cela sont trop souvent balayées par le vent, or, qui n'a pas remarqué ces superbes 
bandes de semis au fond des ornières et compris qu'ils se sont développés là parce que les feuilles se sont accumulées dessus alors qu'elles étaient balayées par le vent ailleurs? De plus, pensons-nous, ce travail ne peut que favoriser la nitrification décrite par M. l'Ingénieur Duchaufour à l'occasion de l'éclairement brusque du sol causé par ta coupe d'ensemencement. Il serait intéressant de favoriser encore le phénomène en épandant avant le travail du sol, et nous comptons l'essayer cette année, une tonne par exemple de scories potassiques I2/I2 à l'hectare.

Il ne faut pas s'imaginer que cette façon culturale est une innovation, c'est seulement la résurrection et la mise au point de façons culturales très anciennes. En effet, l'ordonnance de 1573 le prescrivait déjà formellement en ordonnant que " seront les lieux, ès quels ventes ont été faietes de bois de haute fustaye, pareillement labourez et semez de glands ". M. Potel, dans l'ouvrage déjà cité, donne la nomenclature des opérations effectuées entre i669 et I79I pour la régénération des futaies et dit en particulier: " $3^{\circ}$ Dans le cas où le peuplement naturel était jugé insuffisant, l'ensemencement des coupes était complété artificiellement, aux graines fournies par les arbres réservés on ajoutait d'autres graines répandues après labour du sol "). Il n'y a pas de doute que ces méthodes se sont poursuivies pendant presque tout le siècle dernier et que chaque époque a utilisé les instruments qui lui paraissaient les plus propres à bien effectuer çe travail.

Dans l'Inspection du Mans, à partir de I943, M. le Conservateur Viney, faute de mieux, a remis ces vieux préceptes en vigueur en utilisant une débroussailleuse landaise, mais il est certain que toute la gamme des instruments à disques que nous possédons maintenant, devrait nous donner une supériorité considérable pàr rapport à nos devanciers.

Et ainsi on doit pouvoir aborder, avec déjà le maximum de chances de succès, le cycle des secondaires.

Comme pour le hêtre en Normandie, et plus encore puisqu'il s'agit du chêne qui forme en moyenne les 8/1o du peuplement, elles devront se succéder très rapidement, tous les 2 ou 3 ans, pour que la durée totale de l'ensemencement ne dépasse pas i2 à I 5 ans.

Alors qu'autrefois l'on parlait surtout de dégagements de semis quand ils arrivaient au stade fourré, nous pensons que les coupes, en cours de régénération, doivent absolument être parcourues deux fois par an au minimum. Au printemps ou au début de l'été, îl faut bâtonner les fougères, enlever les morts-bois ou les ronces qui causent aux jeunes plants une concurrence d'où ils sortent toujours battus. L'on a eu à cet égard grand tort, à notre avis, de décrire la ronce comme le berceau du chêne. Remercions-là de nous signaler un sol de bonne qualité où la régénération devra réussir facilement, mais si on la laisse grandir et se propager avant les 
semis, elle les assoiffera et les affamera les tuant sitôt nés. Si elle se développe dans des semis déjà grands, elle n'est pas nuisible, et l'on ne peut d'ailleurs plus pratiquement la supprimer sans abimer les plants. Dans les places à fougères, il faut ainsi souvent passer deux fois. Les produits de ce nettoyage devront être rassemblés en petits tas, au pied des réserves par exemple pour ne pas encombrer le sol partout et empêcher la levée de nouveaux plants.

En fin septembre, les secondaires doivent être à nouveau parcourues pour constater si le sol est encore réceptif, s'il ne s'est pas tassé. recouvert de graminées ou de mousse, et pour y remédier à nouveau par un travail du sol qui là n'affectera déjà plus qu'une partie de la sırface. Si cette partie est relativement importante et facilement définie l'on peut encore faire appel au tracteur avec cover-crop, mais si elle est faible et s'il y a déjà des semis épars qu'il ne faut surtout pas risquer de détruire, c'est la houe à trois dents, à cheval qui donne les meilleurs résultats pour un prix très minime. L'ouvrier qui tient le cheval le guide vers les endroits où le sol est en mauvais état et là où il n'y a pas assez de plants, celui qui tient les mancherons a les yeux fixés au sol et soulève son instrument quand il va buter sur des plants, d'ailleurs ceux-ci avec leur enracinement très vite profond, tiennent bien et glissent le long des dents. Enfin, à l'approche de la définitive, le travail devra être fignolé à la main par crochetage à la houe. Ces travaux devront toujours être exécutés avant la chute des feuilles pour que celles-ci en tombant recouvrent les glands.

Ces opérations, après la deuxième secondaire, devront obligatoirement être accompagnées d'un apport supplémentaire de glands et tout au moins, si la glandée est abondante, ceux-ci devront être répartis sur les endroits non encore régénérés. Les ouvriers, armés de paniers, prendront les glands dans les brosses déjà complètes et les rejetteront sur les emplacements qui en manquent, il est en effet bien connu que ce sont toujours les mêmes pieds qui sont fertiles et les glands qui tombent sur une place déjà bien garnie de semis, en créent en surnombre ou sont perdus. Il est rare, même les années réputées sans glandée, dans l'Ouest, qu'il n'y ait pas un canton dans les grands massifs ou des bordures plus éclairées où l'on ne puisse trouver les quelques quintaux de glands nécessaires à cette opération indispensable.

Ceci a toujours été fait, plus ou moins, mais il faudrait lui redonner le caractère obligatoire qu'il présentait autrefois, car là encore, rien de bien nouveau n'a été imaginé, les conditions particulières des ventes en Bercé et en Perseigne, vers 1808 contenaient en effet les dispositions suivantes: "Les adjudicataires de coupes de futaie ne pourront commencer leur exploitation qu'après avoir jeté et répandu sur la superficie de leur coupe, en présence du garde général' (s'il vous plaît!) et du garde du triage $200 \mathrm{~kg}$ de glands 
et faines par hectare. Les permis d'exploiter ne seront donnés que sur le vu d'un certificat du garde général attestant que l'ensemencement a eu lieu et que les glands et faines étaient de bonne qualité. S'il était reconnu que la coupe fut naturellement et suffisamment repeuplée, l'adjudicataire serait déchargé de cette obligation et il en serait fait mention sur le permis d'exploiter ). Deux cents kilos de glands à l'hectare étant faibles, cette quantité avait été augmentée par la suite.

Nous indiquerons plus loin les prix de revient de tous ces travaux, mais il faut bien s'imaginer que réalisés tous les ans et dès le début de la régénération, ils sont en somme très peu de chose chaque année et donc peu onéreux; si l'on s'est par contre laissé déborder par la nature, leur coût croît très rapidement. Il serait d'ailleurs peut-être possible, c'est à essayer, de diminuer le prix des dégagements annuels, par un apport sur les semis de $50 \mathrm{~kg}$ d'azote pur par hectare. sous forme de nitrate d'ammoniaque $33 \%$ par exemple, qui activant leur croissance, supprimerait 4 ou 5 années de dégagements, cette pratique est constante dans les vergers modernes, même dans la phase où il s'agit seulement de faire grossir les arbres, et il n'y a pas de raison pour que les chênes ne réagissent pas comme des pommiers ou des poiriers...

- Si toutes ces précautions sont consciencieusement observées, l'on peut être à peu près sûr que le semis est installé presque partout, mais il ne suffit pas de le faire naitre, il faut le conserver et il s'agit ici essentiellement des clauses à insérer à cet effet dans les conditions particulières de la vente.

L'un des moyens les plus sûrs d'éviter de gros dégâts est de proscrire les secondaires qui mettent par terre d'un seul coup un matériel trop important, quelle que soit la conscience et le savoir faire des bûcherons, la coupe présentera forcément. l'aspect d'un champ de bataille, et les semis qui avaient provoqué cette coupe trop brutale en seront les victimes. Si les travaux effectués ont bien porté leurs fruits, les secondaires devront se succéder tous les deux où trois ans, mais en ne prenant pas trop de matériel à chaque fois. Il faut signaler cependant que les ennemis des semis sont souvent aussi leurs propres parents. L'on a décrit la nocivité des essences à racine traçante à l'égard de leurs enfants, l'épicéa notamment; tout le monde sait que les gros hêtres présentent les mêmes inconvénierts et les préposés les appellent les " pompiers ", car ils savent bien qu'ils assoifferont jusqu'à ce que mort s'ensuive tout ce qui pousse sous eux, mais les chênes eux-mêmes, dans certains cantons, possèdent un enracinement beaucoup plus superficiel que l'on ne croirait, les culées de chablis le montrent bien, et sous eux, en deux ans, les semis acquis s'étiolent. Ces arbres se manifestent par des hanches très fortes au pied, ils ne s'enfoncent pas en terre, ils s'étalent sur le sol, malgré des houppiers superbes, il faudra souvent les sacrifier rapidement. 


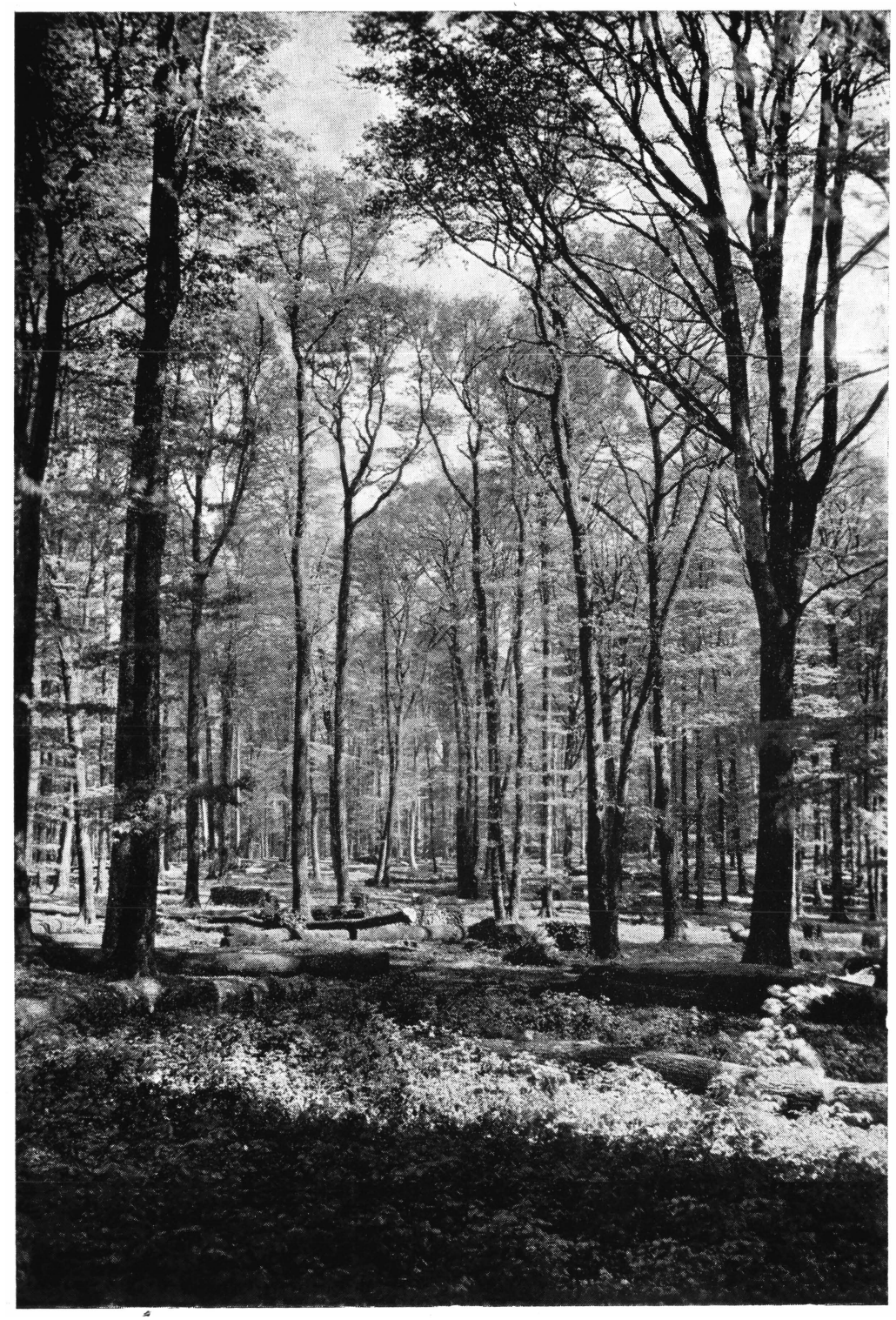

Forêt domaniale de Perseigne.

$2^{\mathrm{n}}$ série - Cánton du Tertre du Buisson - Parcelle 8.

Première Secondaire.

Remarquer au $\mathrm{I}^{\text {er }}$ plan le tapis de semis. Le chauffage a été débardé sur le chemin. Les grumes devraient être parties puisque la feuille est apparue. Les ramiers sont façonnés en bourrées (1948).

(Cliché Hamelin.) 


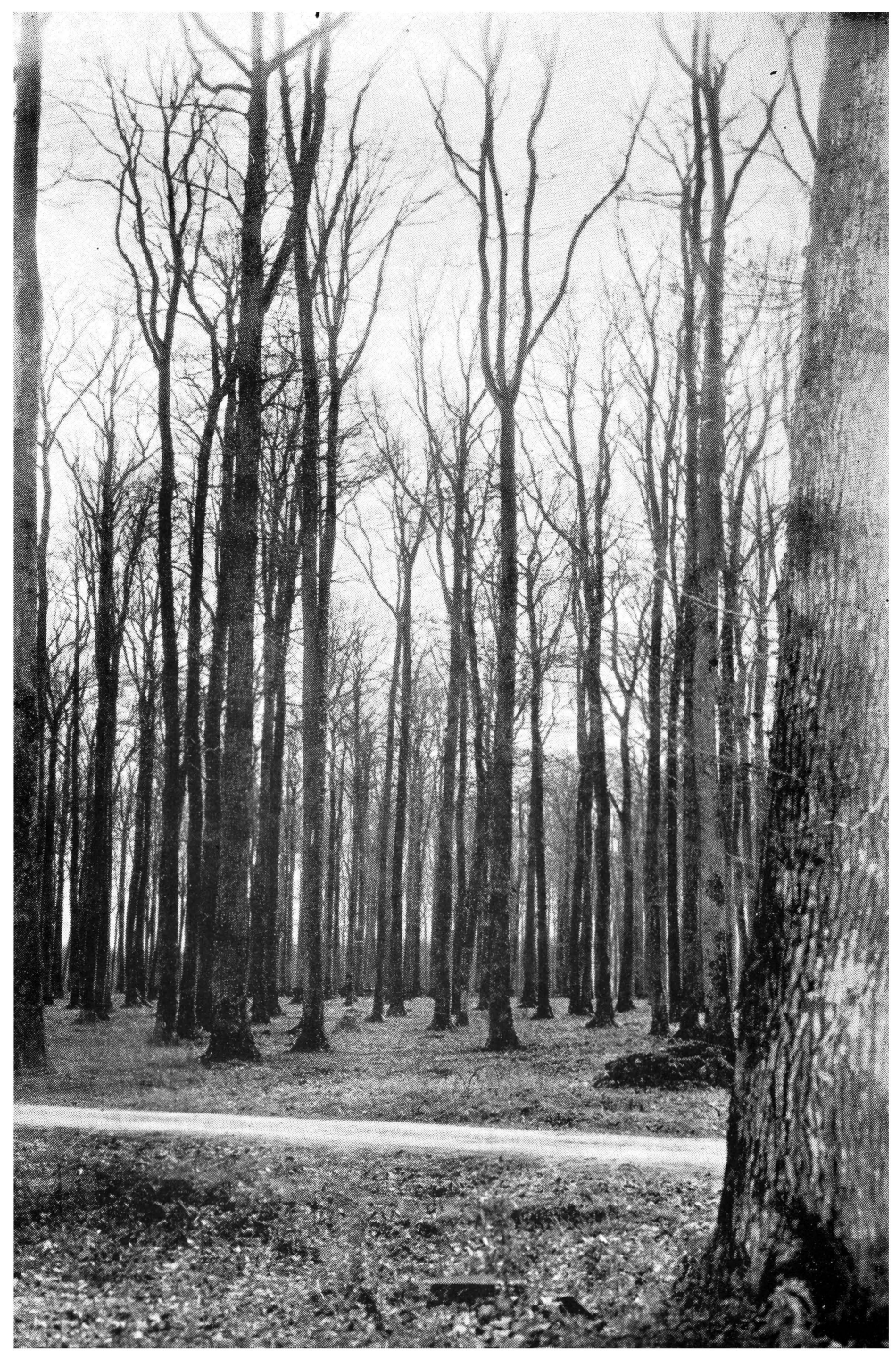

Forêt domaniale de Bercé.

3" série - Canton de la Boulaie - Parcelle A 4.

Coupe d'ensemencement.

Le sol est propre. Les restes d'un premier nettoiement

sont réunis en petits tas.

(Cliché Hamelin.) 
Dans les forêts qui nous intéressent, pour la même raison, les hêtres reproducteurs qui sont indispensables pour que subsiste le mélange optimum devront être choisis parmi les petits diamètres.

En ce qui concerne les exploitations, il s'agit donc d'obtenir que les semis soient le moins possible couchés sous des produits, qu'ils y restent en tout cas le moins longtemps possible, et qu'ils soient au minimum foulés par le débardage.

Ce sont les houppiers, comparés aux troncs, qui couchent et brisent le plus les semis, il faut donc que les clauses particulières imposent l'obligation absolue de façonner les houppiers au fur et à mesure de l'exploitation, que celle-ci ait été ou non précédée de l'éhouppement, et il faut évidemment autant que possible, dans un cas comme dans l'autre, que les houppiers soient dirigés lors de leur chute là où il y a le moins de semis. Mais, lorsque l'on approche de la définitive, et à fortiori lors de celle-ci, des houppiers tombent forcément sur ides taches acquises. Il y a peu de mal s'ils n'y séjournent que quelques jours, les jeunes tiges encore flexibles se redresseront. Il ne faut donc absolument pas admettre un abattage de toute la coupe, puis un façonnage, il faut au contraire que les deux opérations soient simultanées, et il faut encore moins admettre un abattage de toute la coupe, le tronçonnage des produits les plus intéressants et leur sortie immédiate, les tracteurs alors sont obligés de se fraver un chemin au milieu d'un enchevêtrement de ramiers et écrasent tout. Il faut un façonnage à mesure et la sortie immédiate du bois cle chauffage, ainsi les produits les plus nocifs l'auront été pendant un minimum de temps. Les ramilles, qu'il est presque impossible de faire façonner en bourées maintenant, doivent cependlant être débarrassées du parterre de la coupe, elles gêneraient aussi le débarclage et entraveraient tout à fait les travaux décrits précédemment. Elles doivent être sorties dans des tombereaux et brûlées sur les routes ou les chemins constitués dès l'ensemencement. Il est bon de prévoir qu'elles soient là brûlées dans de vieux bas de four à carboniser, le rayonnement du feu est ainsi très réduit et les fourrés avoisinant n'en souffriront pas.

Quant au débardage proprement dit des grumes, il doit être fait aussitôt que possible, dès que le terrain pas trop détrempé ou gelé le permet, et dès que l'adjudicataire aura fait ses découpes et présenté ses lots à ses acheteurs. En tout cas, il faut, sauf temps abominables tout de même exceptionnels. qu'elles soient parties avant que la végétation ne reparte, soit avant le I 5 avril, ainsi les brins couchés peuvent se redresser, ou recépés, ils rejetteront tout de suite.

Le réseau de chemins fixes qui sont prévus dès le début doit grandement faciliter cette opération si délicate qui est capable de démolir en un jour le travail patient de la nature et du forestier pendant plusieurs années. Ces clauses particulières sévères ont été dans l'inspection dụ Mans généralęment bien admises et comprises 
par les adjudicataires sérieux, mais il faut toujours s'y tenir et de très près, et c'est là certaainement l'un des aspects les plus difficiles de la réussite, parce qu'il ne dépend pas que de soi-même ou de son personnel.

Et ainsi la coupe définitive s'achèvera presque à coup sî̀r sur une parcelle peuplée aux 9/1ơ de semis dont le nombre à l'hectare pourra être considérable, de cent mille peut-être, dans lesquels, après l'élimination naturelle des moins forts, l'on pourra avec passion rechercher ces tiges superbes qui alors artificiellement favorisées assureront certainement une production de grande valeur, quelle que soit l'utilisation qui leur sera trouvée lors de leur propre récolte, dans deux cents ans de notre ère, les événements vont vite...

Il reste cependant encore forcément un petit travail de bourrage par plantation à effectuer, car il est à peu près impossible de faire en sorte que les taches de semis soient absolument contiguës. Il sera réalisé avec des hêtres déjà forts de 4 ans, quelques centaines à l'hectare, qui nécessiteront peu de dégagements, rattraperont vite leurs voisins et assureront ainsi le bon élagage des chênes se trouvant en bordure des taches de semis.

Nous nous excusons d'un exposé qui tient de la recette de cuisine ou de la note de service adressée au personnel, en comparaison le: articles hautement scientifiques qui honorent en général cette Revue et en manière de conclusion nous chiffrerons, toujours d'après les connées des faits, le coût moyen de la production ainsi conçue d'un hectare de semis de chênes parsemés de quelques hêtres.

- Remise en état du réseau de drainage - coût évidemment extrêmement variable selon chaque parcelle, en moyenne .......................... 2000

- Travail du sol à l'ensemencement ............. 3000

- Travail du sol pendant les secondaires ........... 2000

-.- Dégagements annuels .................. 10 000

- Apport ou répartition des glands ............. 3000

- Plantation complémentaire ................ 5000

25000

Somme qui, à intérêts composés à $2 \%$ pendant 200 ans, correspond à I 3 I2 $500 \mathrm{~F}$.

Cette somme, comparée au produit escompté, estimé à 4 millions 
par exemple, est encore acceptable alors que les 3570000 correspondants à l'investissement nécessité par une mauvaise régénération à reprendre par plantation est excessive et que dire de la régénération manquée et laissée telle quelle qui ne produira rien ou presque pendant Dieu sait combien de temps!

Ce qu'il faút réaliser au fond, c'est l'heureux mélange des qualités de nos anciens, faites d'observation et de finesse, qui en faisaient d'excellents marteaux, avec la ténacité et le courage de nos très grands anciens qui ne redoutaient pas l'effort toujours répété, pour arriver finalement à dépasser les résultats obtenus au cours des $\mathrm{XVII}^{\mathrm{\theta}}$ et $\mathrm{XVII} \mathrm{I}^{\mathrm{e}}$ siècles qui furent cependant, en bien des matières, ceux de la belle œuvre amoureusement et bien finie.

Ce faisant, nous aurons à peine dépassé les succès du passé sans utiliser, ou presque, les données scientifiques récentes dont certains résultats sont cependant spectaculaires en agriculture, et il faudra bien alors attaquer une nouvelle phase et faire beaucoup micux grâce aux découvertes de là génétique, qui ne s'est pas encore beaucoup occupé du vieux chêne séculaire, grâce peut-être aussi aux engrais et aux traitements chimiques qui trouveront bien là leur utilisation pour débarrasser par exemple nos jeunes plants de l'oïdium ou des chenilles défoliatrices qui, dans ses débuts, retardent bien son développement. Mais ce qui sera difficile en la matière, c'est d'arriver à le faire toujours à peu de frais, car une révolution aussi longue que celle du chêne est incompatible avec un prix de revient trop élevé au départ.

En somme, il faut pour cette culture du chêne, comme pour toutes les autress, méditer toujours les propos d'un célèbre forestier, pourtant rarement cité dans la littérature forestière, Jean de LA Fontaine, que l'on qualifierait en notre époque de " technicien " de la fable, et nous ajouterons du bons sens, et faisait dire à ses fils, par un riche laboureur sentant sa mort prochaine:

" Travaillez, prenez de la peine, C'est le fond qui manque le moins ».

R. LORNE.

\section{La Revue du Bois de mai 1955 publie:}

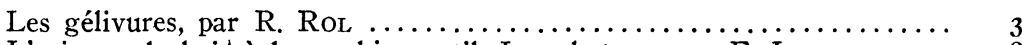

L'usinage du bois à la machine outil. Le rabotage, par E. LEDINot .... 8

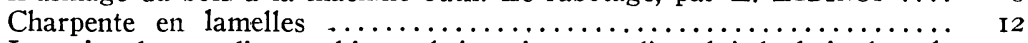

Le point de vue d'un architecte britannique sur l'emploi du bois dans la construction et sa préservation, par C. S. White et H. MARCY ....... I3

Du pétrole dans les Landes de Gascogne, par P. H. Goissard ......... I3

Quelques caractéristiques principales de la sylvicuture et de l'industrie du bois yougoslaves, par B. PEJOSKI $\ldots \ldots \ldots \ldots \ldots \ldots \ldots \ldots \ldots \ldots \ldots \ldots \ldots \ldots$ I 8

Structure cellulaire et propriété mécanique. Le bois d'Epicéa, par A.

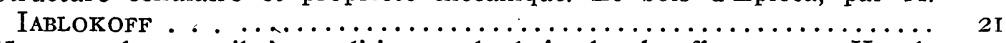

Un nouvel appareil à conditionner le bois de chauffage, par $X$. de

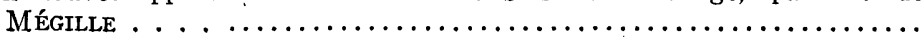

